\title{
Improving the light-harvesting of second generation solar cells with photochemical upconversion
}

\author{
Yuen Yap Cheng ${ }^{1}$, Burkhard Fückel ${ }^{1}$, Tim Schulze ${ }^{1}$, Rowan W. MacQueen ${ }^{1}$, Murad J. Y. \\ Tayebjee ${ }^{1}$, Andrew Danos ${ }^{1}$, Tony Khoury ${ }^{1}$, Raphaël G. C. R. Clady ${ }^{1}$, N. J. Ekins-Daukes ${ }^{2}$, \\ Maxwell J. Crossley ${ }^{1}$, Bernd Stannowski ${ }^{3}$, Klaus Lips ${ }^{4}$ and Timothy W. Schmidt ${ }^{1,5}$ \\ ${ }^{1}$ School of Chemistry, The University of Sydney, NSW 2006, Australia \\ ${ }^{2}$ Department of Physics and the Grantham Institute for Climate Change, Imperial College, \\ London, UK SW7 $2 A Z$ \\ ${ }^{3}$ Competence Centre Thin-Film- and Nanotechnology for Photovoltaics Berlin (PVcomB), \\ Helmholtz-Zentrum Berlin für Materialien und Energie, 12489 Berlin, Germany \\ ${ }^{4}$ Institute for Silicon Photovoltaics, Helmholtz-Zentrum Berlin für Materialien und Energie, \\ 12489 Berlin, Germany \\ ${ }^{5}$ Institute of Photonics and Optical Science, The University of Sydney, NSW 2006, Australia
}

Photovoltaics (PV) offer a solution for the development of sustainable energy sources, relying on the sheer abundance of sunlight: More sunlight falls on the Earth's surface in one hour than is required by its inhabitants in a year. However, it is imperative to manage the wide distribution of photon energies available in order to generate more cost efficient PV devices because single threshold PV devices are fundamentally limited to a maximum conversion efficiency, the Shockley-Queisser (SQ) limit. Recent progress has enabled the production of c-Si cells with efficiencies as high as $25 \%$, ${ }^{1}$ close to the limiting efficiency of $\sim 30 \%$. But these cells are rather expensive, and ultimately the cost of energy is determined by the ratio of system cost and efficiency of the PV device. A strategy to radically decrease this ratio is to circumvent the SQ limit in cheaper, second generation PV devices. One promising approach is the use of hydrogenated amorphous silicon (a-Si:H), where film thicknesses on the order of several $100 \mathrm{~nm}$ are sufficient. Unfortunately, the optical threshold of a-Si:H is rather high $(1.7-1.8 \mathrm{eV})$ and the material suffers from light-induced degradation. Thinner absorber layers in a-Si:H devices are generally more stable than thicker films due to the better charge carrier extraction, but at the expense of reduced conversion efficiencies, especially in the red part of the solar spectrum (absorption losses). Hence for higher bandgap materials, which includes a-Si as well as organic and dye-sensitized cells, the major loss mechanism is the inability to harvest low energy photons.

Ways to overcome the single threshold paradigm by harvesting low energy photons are realised in tandem and multi-junction devices and proposed in intermediate-band solar cells. However, these approaches require reengineering of the solar cell architecture, which comes at increased cost. A method to improve existing solar cell technology is upconversion (UC) of low energy photons. Here, a UC unit is placed behind a bifacial solar cell (SC) such that the low energy photons transmitted by the SC are converted into photons above the bandgap and radiated back into the SC. For UC to operate under incoherent sunlight conditions - in contrast to second harmonic generation, for instance, - an intermediate energy level must be available, preferably long-lived. The UC efficiency increases if the intermediate level has a substructure such that the initially populated state can relax into a lowerlying state which does not couple radiatively to the initial state of the excitation.

In photochemical UC, usually afforded by dye-sensitised triplet-triplet annihilation (TTA), this is the case. Sensitizer molecules are promoted from the $\mathrm{S}_{0}$ singlet ground state to $\mathrm{S}_{1}$ by absorbing low energy photons. Intersystem crossing results in a non-adiabatic transition to the long-lived $\mathrm{T}_{1}$ triplet state, satisfying the aforementioned energy sacrifice requirements. Subsequent triplet energy transfer (TET) to emitter molecules (in excess) produces a population of emitter triplets which then undergo triplet-triplet annihilation (TTA) to produce $\mathrm{S}_{1} \rightarrow \mathrm{S}_{0}$ emission at a higher energy than the photons absorbed. In this contribution, we report the first integrated a-Si:H/TTA-UC photovoltaic device.

Organic Photovoltaics XIII, edited by Zakya H. Kafafi, Christoph J. Brabec, Paul A. Lane, Proc. of SPIE Vol. 8477, 84770X - (C) 2012 SPIE - CCC code: 0277-786X/12/\$18 - doi: 10.1117/12.945217 

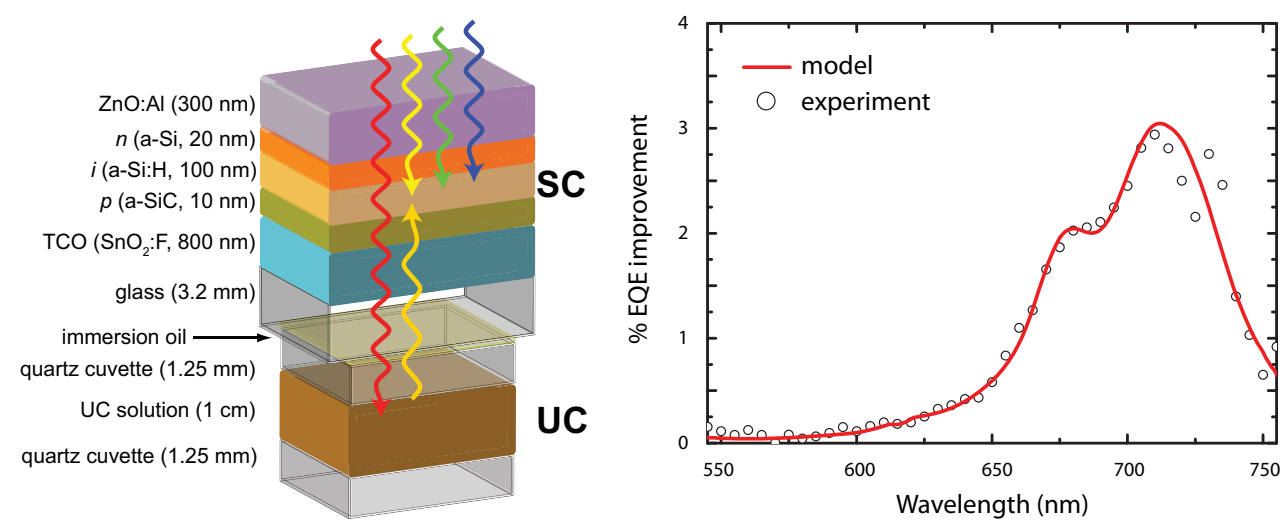

Figure 1: (Left) The a-Si:H cell invoked in this study, optically contacted to a photochemical upconvertor. (Right) The ratio of EQE curves measured with the upconvertor operative and inoperative. The absorption peak of the sensitizer molecule is evident in the relative efficiency increase.

In Fig. 1, we show a p-i-n a-Si:H solar cell, augmented with an upconversion solution. The EQE curve is measured for the device, with and without a continuous excitation laser overlapping the chopped, monochromated probe. The ratio of the EQE curves thus obtained reveals the efficacy of the upconversion. As can be seen in Fig. 1, a 3\% enhancement of the EQE is obtained in the $650-700 \mathrm{~nm}$ region, at an equivalence of 19 suns. We can compare to conventional rare-earth (RE) based upconversion by calculating the expected enhancement under the concentrated AM1.5 spectrum. Our best system as of February 2012 generated $1.3 \times 10^{-4} \mathrm{~mA} \mathrm{~cm}{ }^{-2} \mathrm{sun}^{-2}$, while the $2010 \mathrm{RE}$ results of de Wild et al., using thicker a:Si:H cells, generated only $6.6 \times 10^{-7} \mathrm{~mA} \mathrm{~cm}{ }^{-2} \mathrm{sun}^{-2}{ }^{3}$ As such, the first demonstration of photochemical upconversion was 200 times more efficient than rare-earth phosphors. Addition of a reflective back-layer to the device increases this figure of merit still further. The back-scatterer not only reflects more upconverted light into the solar cell, but also increases the light harvesting by scattering photons at the rear of the device (Fig. 2). With this in place, we obtained $3 \times 10^{-4} \mathrm{~mA} \mathrm{~cm}^{-2} \mathrm{sun}^{-2}{ }^{4}$. Through better matching of the solar cell to the upconvertor, we arrive at the latest result of $1 \times 10^{-3} \mathrm{~mA} \mathrm{~cm}^{-2} \mathrm{sun}^{-2}$. The chronology of the improving Figure of Merit is shown in Fig. 3.

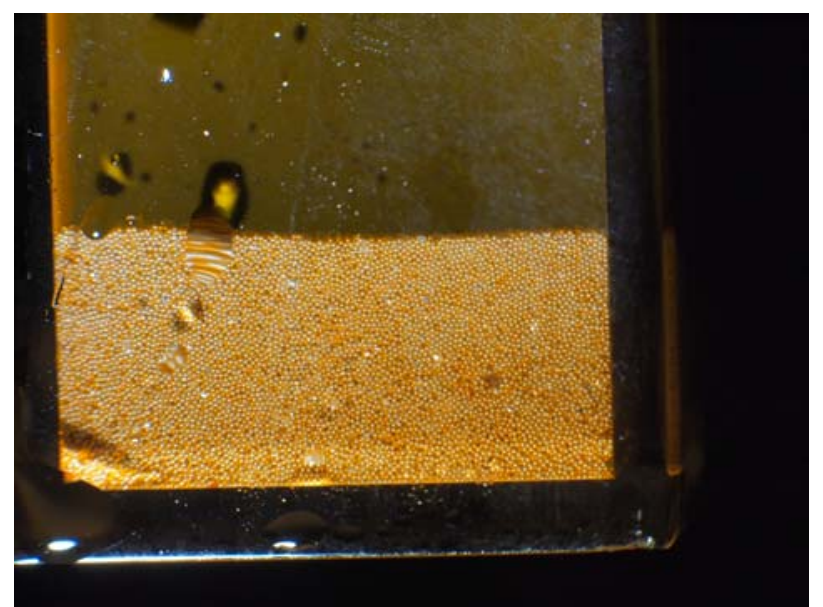

Figure 2: The back-scatter in the upconversion cuvette. The cuvette is filled with $100 \mu \mathrm{m}$ silver-coated glass spheres. 


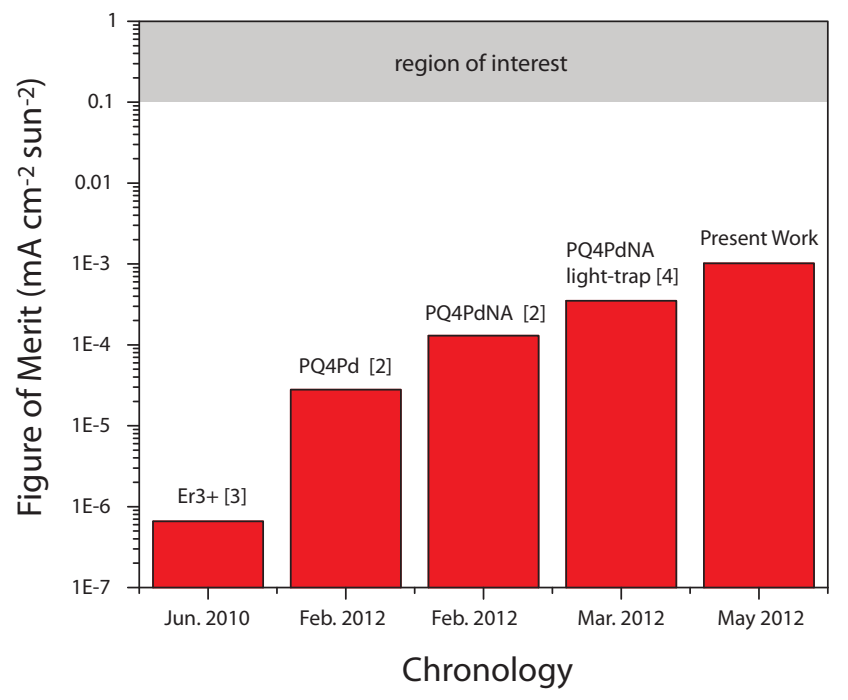

Figure 3: Evolution of the Figure of Merit for upconvertor assisted a-Si:H solar cells.

Yet, improvements can be achieved by mixing sensitizer molecules to broaden the absorption range, or the use of plasmonics to spatially localize the excitation light to create higher local triplet densities. But, the biggest improvement will come from reengineering the UC unit at the molecular scale. TTA-UC at low excitation is generally limited by the TTA kinetics, where the triplet decay of the emitter is faster than the encounter with another triplet emitter. Hence, faster diffusion of and/or shorter distance between the emitter triplets increases the amount of second order decay and thus the UC efficiency. The average distance between the triplet source under the current conditions is $17 \mathrm{~nm}$. If this can be reduced by a factor of $4-5$, then the triplet concentration will be increased hundredfold, and the TTA efficiency is boosted into the region of interest.

\section{References}

[1] Green, M. A., "The path to 25\% silicon solar cell efficiency: History of silicon cell evolution" Prog. Photovolt. Res. Appl. $17(3), 183-189(2009)$

[2] Cheng, Y. Y., Fückel, B., MacQueen, R. W., Khoury, T., Clady, R. G. C. R., Schulze, T. F., Ekins-Daukes, N. J., Crossley, M. J., Stannowski, B., Lips, K. and Schmidt, T. W., "Improving the light-harvesting of amorphous silicon solar cells with photochemical upconversion" Energy Environ. Sci. 5, 6953-6959 (2012).

[3] de Wild, J., Rath, J. K., Meijerink, A., van Sark, W. G. J. H. M. and Schropp, R. E. I. "Enhanced near-infrared response of aSi:H solar cells with $\beta$-NaYF4: $\mathrm{Yb}^{3+}(18 \%), \mathrm{Er}^{3+}(2 \%)$ upconversion phosphors" Sol. Energy Mater. Sol. Cells 94(12), 2395-2398 (2010).

[4] Schulze, T. F., Cheng, Y. Y., Fückel, B., MacQueen, R. W., Danos, A., Davis, N. J. L. K., Tayebjee, M. J. Y., Khoury, T., Clady, R. G. C. R., Ekins-Daukes, N. J., Crossley, M. J., Stannowski, B., Lips, K. and Schmidt, T. W., "Photochemical Upconversion Enhanced Solar Cells: Effect of a Back Reflector" Aust. J. Chem. 65, (2012) doi:10.1071/CH12117 